PROCEEDINGS OF THE

AMERICAN MATHEMATICAL SOCIETY

Volume 135, Number 11, November 2007, Pages 3631-3640

S 0002-9939(07)08969-1

Article electronically published on August 7, 2007

\title{
A CRITERION \\ FOR THE LOGARITHMIC DIFFERENTIAL OPERATORS TO BE GENERATED BY VECTOR FIELDS
}

\author{
MATHIAS SCHULZE
}

(Communicated by Michael Stillman)

\begin{abstract}
We study divisors in a complex manifold in view of the property that the algebra of logarithmic differential operators along the divisor is generated by logarithmic vector fields. We give

- a sufficient criterion for the property,

- a simple proof of F.J. Calderón-Moreno's theorem that free divisors have the property,

- a proof that divisors in dimension 3 with only isolated quasi-homogeneous singularities have the property,

- an example of a nonfree divisor with nonisolated singularity having the property,

- an example of a divisor not having the property, and

- an algorithm to compute the V-filtration along a divisor up to a given order.
\end{abstract}

\section{LOGARITHMIC COMPARISON THEOREM FOR FREE DIVISORS}

Let $X$ be a complex manifold of dimension $n \geq 2, \mathcal{O}$ the ring holomorphic functions on $X$, and $\Omega^{\bullet}$ the complex of holomorphic differential forms. Grothendieck's Comparison Theorem states that the De Rham system $\mathcal{O}$ is regular Meb89, Thm. 2.3.4]. This is equivalent to the fact that, for any divisor $D \subset X$, the natural morphism

$$
\Omega^{\bullet}(* D)=\mathrm{DR}(\mathcal{O}(* D)) \longrightarrow \mathrm{R} i_{*} i^{-1} \mathrm{DR}(\mathcal{O})=\mathrm{R} i_{*} \mathbb{C}_{U},
$$

where $i$ is the inclusion $U=X \backslash D \subset X$, a quasi-isomorphism. Let $\Omega^{\bullet}(\log D) \subset$ $\Omega^{\bullet}(* D)$ be the subcomplex of logarithmic differential forms along $D$ [Sai80, Def. 1.2]. The above statement raises the question whether the inclusion $\Omega^{\bullet}(\log D) \subset \Omega^{\bullet}(* D)$ is also a quasi-isomorphism. That is: Can one compute the cohomology of the complement of $D$ by logarithmic differential forms along $D$ ? This turns out to be a property of $D$ called the logarithmic comparison theorem or simply LCT. It is an open problem to characterize the divisors for which LCT holds.

Received by the editors September 16, 2005 and, in revised form, September 2, 2006.

2000 Mathematics Subject Classification. Primary 32C38, 13A30.

Key words and phrases. Free divisor, hyperplane arrangement, logarithmic differential operator, symmetric algebra, V-filtration.

The author is grateful to M. Granger for many valuable discussions and comments and to F.J. Castro-Jiménez, L. Narváez-Macarro, and J.M. Ucha-Enríquez for explaining their results and ideas. 
Let $\Theta=\operatorname{Der}_{\mathbb{C}}(\mathcal{O})$ be the $\mathcal{O}$-module of holomorphic vector fields on $X$ and $\operatorname{Der}(\log D) \subset \Theta$ be the $\mathcal{O}$-submodule of logarithmic differential operators along $D$ [Sai80, Def. 1.4]. A divisor $D$ is called free if $\operatorname{Der}(\log D)$ is a locally free $\mathcal{O}$ module. Let $\mathcal{D}$ be the $\mathcal{O}$-algebra of differential operators on $X$ with holomorphic coefficients, and let $F$ be the increasing filtration on $\mathcal{D}$ by the order of differential operators. Let $\mathcal{V}^{D}$ be the V-filtration along $D$ on $\mathcal{D}$ as defined in Section 2 such that $\mathcal{V}_{0}^{D}=\mathcal{D}(\log D)$ is the $\mathcal{O}$-algebra of logarithmic differential operators along $D$. F.J. Calderón-Moreno CM99, Thm. 1] proves that, for a free divisor $D, \mathcal{V}_{0}^{D}$ is generated by vector fields, that is, $\mathcal{V}_{0}^{D}=\mathcal{O}[\operatorname{Der}(\log D)]$. Let $S_{D}$ be the decreasing filtration on $\mathcal{D}$ which is locally defined by $S_{D}^{k}=f^{k} \cdot \mathcal{D}$ where $f \in \mathcal{O}$ such that $D=(f)$. By Corollary 3, the induced filtration $S_{D}$ on $\mathcal{V}_{0}^{D}$ defined by $S_{D}^{k} \mathcal{V}_{0}^{D}=$ $\mathcal{V}_{0}^{D} \cap\left(f^{k} \cdot \mathcal{D}\right)$ reflects the embeddings $\mathcal{V}_{k}^{D} \subset \mathcal{D}$. If $\mathcal{V}_{0}^{D}=\mathcal{O}[\operatorname{Der}(\log D)]$, then $\left(\mathcal{V}_{0}^{D}, S_{D}\right)$ is a filtered $\left(\mathcal{V}_{0}^{D}, \mathcal{V}^{D}\right)$-module.

F.J. Calderón-Moreno and L. Narváez-Macarro [CMNM05, Cor. 4.2] prove that LCT holds for a free divisor $D$ if and only if the complex

$$
\mathcal{D} \stackrel{\mathrm{L}}{\otimes}_{\mathcal{D}(\log D)} \mathcal{O}(D)=\mathcal{D} \otimes_{\mathcal{D}(\log D)} \operatorname{Sp}_{\mathcal{D}(\log D)}^{\cdot(\mathcal{O}(D))}
$$

is concentrated in degree 0 and the natural multiplication morphism

$$
\mathcal{D} \otimes_{\mathcal{D}(\log D)} \mathcal{O}(D) \stackrel{\epsilon_{D}}{\longrightarrow} \mathcal{O}(* D)
$$

is an isomorphism. Injectivity of $\epsilon_{D}$ is locally equivalent to $\operatorname{Ann}_{\mathcal{D}}\left(\frac{1}{f}\right)$ being generated by operators of order 1 where $f \in \mathcal{O}$ such that $D=(f)$. For any divisor $D, \mathrm{~T}$. Torrelli proves that the latter condition already implies surjectivity of $\epsilon_{D}$ [Tor04, Prop. 1.3] and conjectures that it is even equivalent to LCT [Tor04, Conj. 1.11]. A problem in verifying this conjecture for a free divisor $D$ consists in $\mathcal{D} \otimes_{\mathcal{D}(\log D)} \operatorname{Sp}_{\mathcal{D}(\log D)}(\mathcal{O}(D))$ not being $F$-strict in general [CM99, Rem. 4.2.4]. So grading by $F$ does not reduce the problem to a commutative one. But both properties of $D$ in question can be characterized in terms of $S_{D}$-strictness: On the one hand, exactness of $\mathcal{D} \otimes_{\mathcal{D}(\log D)} \operatorname{Sp}_{\mathcal{D}(\log D)}(\mathcal{O}(D))$ in degree $k$ is equivalent to $S_{D}$-strictness of the differential of $\operatorname{Sp}_{\mathcal{D}(\log D)}^{\bullet}(\mathcal{O}(D))$ from degree $k-1$ to degree $k$. On the other hand, injectivity of $\epsilon_{D}$ is equivalent to $S_{D}$-strictness of the last differential of $\mathcal{D} \otimes_{\mathcal{D}(\log D)} \operatorname{Sp}_{\mathcal{D}(\log D)}(\mathcal{O}(D))$.

A solution of the LCT problem seems to require a deeper understanding of the V-filtration in general. There are many questions:

- What are the properties of the $\mathcal{V}_{k}^{D}$ ?

- When is $\mathcal{V}_{0}^{D}$ generated by vector fields?

- When is $\mathcal{V}_{0}^{D}$ locally finitely generated?

- What are the properties of the embeddings $\mathcal{V}_{k}^{D} \subset \mathcal{D}$ ?

We shall approach the first two questions in this article.

\section{V-FILTRATION ALONG SUBVARIETIES AND DIVISORS}

Let $Y \subset X$ be a subvariety in $X$ and let $\mathcal{I} \subset \mathcal{O}$ be its ideal. The $\mathrm{V}$-filtration $\mathcal{V}^{Y}$ along $Y$ is the increasing filtration on $\mathcal{D}$ defined by

$$
\mathcal{V}_{k}^{Y}=\left\{P \in \mathcal{D} \mid \forall l \in \mathbb{Z}: P\left(\mathcal{I}^{l}\right) \subset \mathcal{I}^{l-k}\right\}
$$


for all $k \in \mathbb{Z}$. We shall omit the index $Y$ if it is clear from the context. Clearly $\mathcal{V}_{k} \cdot \mathcal{V}_{l} \subset \mathcal{V}_{k+l}$ for all $k, l \in \mathbb{Z}$. Hence $\mathcal{V}_{0}$ is an $\mathcal{O}$-algebra and $\mathcal{V}_{k}$ is a $\mathcal{V}_{0}$-module for all $k \in \mathbb{Z}$.

Example 1. Let $x_{1}, \ldots, x_{m}, y_{1}, \ldots, y_{n}$ be coordinates on $X=\mathbb{C}^{m+n}$.

(1) For the submanifold $Y=\{y=0\}$,

$$
\mathcal{V}_{k}^{Y}=\left\{P=\sum_{j_{1}-i_{1}+\cdots+j_{n}-i_{n} \leq k} P_{i, j}\left(x, \partial_{x}\right) y_{1}^{i_{1}} \partial_{y_{1}}^{j_{1}} \cdots y_{n}^{i_{n}} \partial_{y_{n}}^{j_{n}} \in \mathcal{D}\right\} .
$$

(2) For the normal crossing divisor $D=\left(y_{1} \cdots y_{n}\right)$,

$$
\mathcal{V}_{k}^{D}=\left\{P=\sum_{j_{1}-i_{1}, \ldots, j_{n}-i_{n} \leq k} P_{i, j}\left(x, \partial_{x}\right) y_{1}^{i_{1}} \partial_{y_{1}}^{j_{1}} \cdots y_{n}^{i_{n}} \partial_{y_{n}}^{j_{n}} \in \mathcal{D}\right\} .
$$

Denote the complement of the singularities of $Y$ by

$$
U_{Y}=X \backslash \operatorname{Sing}(Y) \stackrel{i_{Y}}{\longrightarrow} X .
$$

We shall omit the index $Y$ if it is clear from the context. The $\mathrm{V}$-filtration along a divisor has a special property.

Proposition 2. Let $D \subset X$ be a divisor. Then $\mathcal{V}^{D}=\left(i_{D}\right)_{*} i_{D}^{-1} \mathcal{V}^{D}$.

Proof. We may assume that $D=(f)$ for some $f \in \mathcal{O}$ by the local nature of the statement. Since $\mathcal{V}_{k} \subset \mathcal{D}$ and $\mathcal{D}$ is a locally free $\mathcal{O}$-module,

$$
i_{*} i^{-1} \mathcal{V}_{k} \subset i_{*} i^{-1} \mathcal{D}=\mathcal{D}
$$

Since $\mathcal{O} \cdot f^{l-k}$ is a free $\mathcal{O}$-module, $P \in i_{*} i^{-1} \mathcal{V}_{k}$ implies that

$$
P\left(g \cdot f^{l}\right) \in i_{*} i^{-1}\left(\mathcal{O} \cdot f^{l-k}\right)=\mathcal{O} \cdot f^{l-k}
$$

for all $g \in \mathcal{O}$ and $l \in \mathbb{Z}$ and hence $P \in \mathcal{V}_{k}$.

Corollary 3. Let $D=(f) \subset X$ with $f \in \mathcal{O}$ a divisor. Then

$$
\mathcal{V}_{k}= \begin{cases}f^{-k} \mathcal{V}_{0}, & k \leq 0, \\ f^{-k}\left(\mathcal{V}_{0} \cap f^{k} \mathcal{D}\right), & k \geq 1\end{cases}
$$

Proof. The equalities in question hold on $U_{D}$ by Example 1(2) and hence on $X$ by Proposition 2

Denote the symbol map for $F$ by

$$
\mathcal{D} \stackrel{\sigma}{\longrightarrow} \operatorname{gr}^{F} \mathcal{D} \text {. }
$$

The decomposition $F_{1} \mathcal{D}=\mathcal{O} \oplus \Theta$ defines the $\mathcal{O}$-module $\operatorname{Der}(\log Y) \subset \Theta$ of logarithmic vector fields along $Y$ by

$$
F_{1} \mathcal{V}_{0}=\mathcal{O} \oplus \operatorname{Der}(\log Y)
$$

This definition simplifies to

$$
\operatorname{Der}(\log Y)=\{\theta \in \Theta \mid \theta(\mathcal{I}) \subset \mathcal{I}\}
$$

by the Leibniz rule and implies involutivity of $\operatorname{Der}(\log Y)$, that is,

$$
[\operatorname{Der}(\log Y), \operatorname{Der}(\log Y)] \subset \operatorname{Der}(\log Y) .
$$


Example 4. Let $x_{1}, \ldots, x_{m}, y_{1}, \ldots, y_{n}$ be coordinates on $X=\mathbb{C}^{m+n}$.

(1) For the submanifold $Y=\{y=0\}$,

$$
\operatorname{Der}(\log D)=\mathcal{O}\left\langle\partial_{x_{1}}, \ldots, \partial_{x_{m}}\right\rangle+\mathcal{O}\left\langle y_{i} \partial_{y_{j}} \mid 1 \leq i, j \leq n\right\rangle .
$$

(2) For the normal crossing divisor $D=\left(y_{1} \cdots y_{n}\right)$,

$$
\operatorname{Der}(\log D)=\mathcal{O}\left\langle\partial_{x_{1}}, \ldots, \partial_{x_{m}}, y_{1} \partial_{y_{1}}, \ldots, y_{n} \partial_{y_{n}}\right\rangle .
$$

Let $\mathcal{O}[\operatorname{Der}(\log Y)] \subset \mathcal{D}$ be the image of the tensor algebra

$$
\mathrm{T}_{\mathbb{C}} \operatorname{Der}(\log Y) \stackrel{\gamma_{Y}}{\longrightarrow} \mathcal{D} .
$$

Then at least $\mathcal{O}[\operatorname{Der}(\log Y)] \subset \mathcal{V}_{0}^{Y}$.

Corollary 5. Let $D \subset X$ be a divisor. Then $\mathcal{V}_{0}^{D}=\mathcal{O}[\operatorname{Der}(\log D)]$ if and only if $\mathcal{O}[\operatorname{Der}(\log D)]=\left(i_{D}\right)_{*} i_{D}^{-1} \mathcal{O}[\operatorname{Der}(\log D)]$.

Proof. By Examples 1 and $4, \mathcal{V}_{0}^{D}=\mathcal{O}[\operatorname{Der}(\log D)]$ on $U_{D}$. Hence the claim follows from Proposition 2.

A divisor $D \subset X$ is called free if $\operatorname{Der}(\log D)$ is a locally free $\mathcal{O}$-module. By K. Saito [Sai80, Cor. 1.7], $\operatorname{Der}(\log D)$ is reflexive and hence all divisors in dimension $n=2$ are free. By Example 4 (2), normal crossing divisors are free. In particular, any divisor $D$ is free on $U_{D}$.

F.J. Calderón-Moreno [CM99, Thm. 1] proves that $\mathcal{V}_{0}^{D}=\mathcal{O}[\operatorname{Der}(\log D)]$ for a free divisor. We give a simple proof of this result.

Corollary 6. Let $D \subset X$ be a free divisor. Then $\mathcal{V}_{0}^{D}=\mathcal{O}[\operatorname{Der}(\log D)]$.

Proof. By Lemma 7 and grading by $F, \mathcal{O}[\operatorname{Der}(\log D)]$ is a locally free $\mathcal{O}$-module and hence Corollary 5 applies.

Lemma 7. Let $R$ be a domain and let $P_{1}, \ldots, P_{n} \in R \cdot T_{1} \oplus \cdots \oplus R \cdot T_{n}=R^{n}$ be $R$-linearly independent. Then $R\left[P_{1}, \ldots, P_{n}\right] \subset R\left[T_{1}, \ldots, T_{n}\right]$ is a polynomial ring.

Proof. Write $P_{i}=\sum_{j} p_{i, j} T_{j}$ with $p_{i, j} \in R$. Then by assumption $p=\operatorname{det}\left(p_{i, j}\right) \neq 0$ and hence $R_{p}\left[P_{1}, \ldots, P_{n}\right]$ is a polynomial ring. Since $R$ is a domain, $R \longrightarrow R_{p}$ is injective and hence $R\left[P_{1}, \ldots, P_{n}\right]$ is a polynomial ring.

In general it is not clear if, or under which conditions, $\mathcal{V}_{0}^{Y}$ is a locally finite $\mathcal{O}$ algebra. Even to compute $F_{k} \mathcal{V}_{0}^{Y}$ is a problem since the definition involves infinitely many conditions. The following result allows one to compute $F_{k} \mathcal{V}_{0}^{D}$ algorithmically.

Proposition 8. Let $x_{1}, \ldots, x_{n}$ be coordinates on $X=\mathbb{C}^{n}$. Let $D=(f) \subset X$ with $f \in \mathcal{O}$ be a divisor. Then, for $P \in F_{d} \mathcal{D}, P \in \mathcal{V}_{k}^{D}$ if and only if

$$
\forall \alpha \in \mathbb{N}^{n}, l \in \mathbb{N}:|\alpha|+l \leq d \Rightarrow P\left(x^{\alpha} f^{l}\right) \in \mathcal{O} \cdot f^{l-k} .
$$

Proof. Let $0 \neq P \in F_{d} \mathcal{D}$ and assume that condition (1) holds. For $l \in \mathbb{N}$, the vector space $\mathbb{C}\left[x_{1}, \ldots, x_{n}\right]_{\leq d-l}$ is invariant under $x \mapsto A x+a$ for $a \in \mathbb{C}^{n}$ and $A \in \mathrm{GL}_{n}(\mathbb{C})$. Hence, at a smooth point $y$ of $D$, condition (1) holds for coordinates $x_{1}, \ldots, x_{n}$ at $y$ such that $\partial_{x_{n}}(f)(y) \neq 0$. Then $y_{1}, \ldots, y_{n-1}, t=x_{1}, \ldots, x_{n-1}, f$ are coordinates at $y$ such that

$$
\forall \beta \in \mathbb{N}^{n-1}, l \in \mathbb{N}:|\beta|+l \leq d \Rightarrow P_{y}\left(y^{\beta} t^{l}\right) \in \mathcal{O}_{y} \cdot t^{l-k} .
$$


Write $P_{y}=\sum_{|\beta|+l \leq d} p_{\beta, l} \partial_{y}^{\beta} \partial_{t}^{l}$ with $p_{\beta, l} \in \mathcal{O}_{y}$ and choose $\gamma \in \mathbb{N}^{n-1}$ and $m \in \mathbb{N}$ such that $|\gamma|+m$ is minimal with $p_{\gamma, m} \neq 0$. Then

$$
\gamma ! m ! p_{\gamma, m}=P\left(y^{\gamma} t^{m}\right) \in \mathcal{O}_{y} \cdot t^{m-k},
$$

and hence $p_{\gamma, m} \partial_{y}^{\gamma} \partial_{t}^{m} \in \mathcal{V}_{k, y}$ by Example1(2). By increasing induction on $|\gamma|+m$, this implies that $P_{y} \in \mathcal{V}_{k, y}$ for all $y \in U_{D}$ and hence $P \in \mathcal{V}_{k}$ by Proposition 2

Example 9. Let $x, y, z$ be coordinates on $\mathbb{C}^{3}$ and

$$
f=x y z(x+y+z)(x+2 y+3 z) .
$$

Then $D=(f) \subset \mathbb{C}^{3}$ is a central generic hyperplane arrangement. Let

$$
Q=(x+y+z)(x+2 y+3 z)\left(3 z y^{2} \partial_{y}^{2}+(x+4 y-3 z) y z \partial_{y} \partial_{z}-4 y z^{2} \partial_{z}^{2}\right) .
$$

Then $Q \in F_{2} \mathcal{V}_{0}^{D}$ by a Singular GPS05] computation using Proposition 8 , We shall see in Example 13 that $Q \notin F_{2} \mathcal{O}[\operatorname{Der}(\log D)]$.

There is another special property of the $\mathrm{V}$-filtration along a divisor.

Proposition 10. Let $D \subset X$ be a divisor. Then $\operatorname{depth}_{x}\left(\mathcal{V}_{k}^{D}\right) \geq 2$ for all $x \in X$ and $k \in \mathbb{Z}$.

Proof. Let $x \in X$ and $D_{x}=(f)$ with $f \in \mathcal{O}_{x}$. Since $\mathcal{O}_{x}$ is torsion free and $\operatorname{depth}\left(\mathcal{O}_{x}\right) \geq 2$, there is an $\mathcal{O}_{x}$-sequence $a_{1}, a_{2} \in \mathfrak{m}_{x}$ such that $a_{1}$ is different from all irreducible factors of $f$. Let $P \in \mathcal{V}_{k, x}$ with $a_{2} \cdot P \in a_{1} \cdot \mathcal{V}_{k, x} \subset a_{1} \cdot \mathcal{D}_{x}$. Then $P \in a_{1} \cdot \mathcal{D}_{x}$ since $\mathcal{D}_{x}$ is a free $\mathcal{O}_{x}$-module. But $P\left(g \cdot f^{l}\right) \in \mathcal{O}_{x} \cdot f^{l-k}$ implies that $\left(a_{1}^{-1} \cdot P\right)\left(g \cdot f^{l}\right) \in \mathcal{O}_{x} \cdot f^{l-k}$ by the choice of $a_{1}$ for all $g \in \mathcal{O}$ and $l \in \mathbb{Z}$, and hence $P \in a_{1} \cdot \mathcal{V}_{0, x}$. Then $a_{1}, a_{2} \in \mathfrak{m}_{x}$ is a $\mathcal{V}_{k, x}$-sequence, and hence $\operatorname{depth}_{x}\left(\mathcal{V}_{k}\right) \geq 2$.

\section{Symmetric Algebra of LOGARITHMiC VeCtor FIELDS}

The condition in Corollary 5 is difficult to verify in general. Therefore we focus on a case in which it still holds after grading by $F$. Consider the commutative diagram of graded algebras

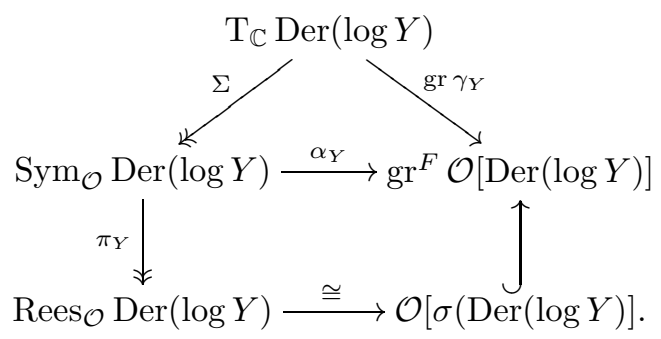

Lemma 11. If $\alpha_{Y}$ is an isomorphism, then

$$
\operatorname{Sym}_{\mathcal{O}} \operatorname{Der}(\log Y)=\left(i_{Y}\right)_{*} i_{Y}^{-1} \operatorname{Sym}_{\mathcal{O}} \operatorname{Der}(\log Y)
$$

implies that $\mathcal{O}[\operatorname{Der}(\log Y)]=\left(i_{Y}\right)_{*} i_{Y}^{-1} \mathcal{O}[\operatorname{Der}(\log Y)]$. 
Proof. The following diagram is commutative.

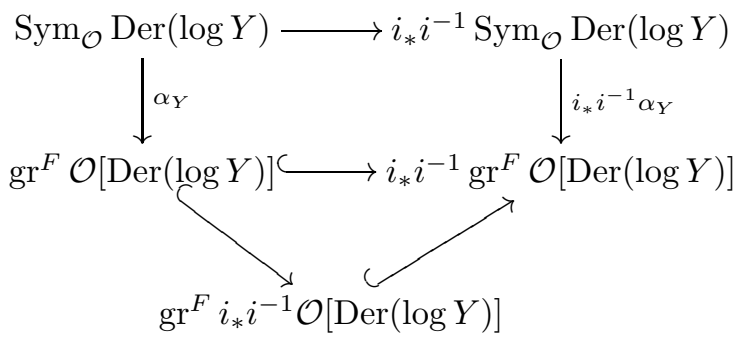

Then the claim follows by induction on $\operatorname{deg}(P)$ for $P \in i_{*} i^{-1} \mathcal{O}[\operatorname{Der}(\log Y)]$.

Lemma 12. $\alpha_{Y}$ is an isomorphism if and only if $\pi_{Y}$ is injective.

Proof. Assume that $\pi_{Y}$ is injective. An element of $\operatorname{gr}^{F} \mathcal{O}[\operatorname{Der}(\log Y)]$ is of the form $\sigma\left(\gamma_{Y}(P)\right)$ where $P \in \mathrm{T}_{\mathbb{C}} \operatorname{Der}(\log Y)$. Write $P=P_{0} \oplus \cdots \oplus P_{d}$ where $d=\operatorname{deg}(P)$. If $\sigma\left(\gamma_{Y}(P)\right) \notin \operatorname{im} \alpha_{Y}$, then $\left(\operatorname{gr} \gamma_{Y}\right)\left(P_{d}\right)=\left(\operatorname{gr} \gamma_{Y}\right)(P)=0$ and hence $P_{d} \in \operatorname{ker} \Sigma$ by injectivity of $\pi_{Y}$. By the definition of $\operatorname{Sym}_{\mathcal{O}}$, this implies that $P_{d}$ is in the twosided ideal generated by the relations $\xi \otimes \eta-\eta \otimes \xi$ and $\xi \otimes(a \eta)-(a \xi) \otimes \eta$ where $\xi, \eta \in \operatorname{Der}(\log Y)$ and $a \in \mathcal{O}$. But

$$
\gamma_{Y}(\xi \otimes \eta-\eta \otimes \xi)=\xi \eta-\eta \xi=[\xi, \eta] \in \operatorname{Der}(\log Y)
$$

by involutivity of $\operatorname{Der}(\log Y)$, and

$$
\gamma_{Y}(\xi \otimes(a \eta)-(a \xi) \otimes \eta)=\xi a \eta-a \xi \eta=[\xi, a] \eta=\xi(a) \eta \in \operatorname{Der}(\log Y) .
$$

This means that

$$
\begin{aligned}
\xi \otimes \eta-\eta \otimes \xi & \equiv[\xi, \eta] \bmod \operatorname{ker} \gamma_{Y}, \\
\operatorname{deg}(\xi \otimes \eta-\eta \otimes \xi) & >\operatorname{deg}([\xi, \eta]), \\
\xi \otimes(a \eta)-(a \xi) \otimes \eta & \equiv \xi(a) \eta \bmod \operatorname{ker} \gamma_{Y}, \\
\operatorname{deg}(\xi \otimes(a \eta)-(a \xi) \otimes \eta) & >\operatorname{deg}(\xi(a) \eta) .
\end{aligned}
$$

Hence $\gamma_{Y}(P)=\gamma_{Y}\left(P^{\prime}\right)$ and $\operatorname{deg}(P)<\operatorname{deg}\left(P^{\prime}\right)$ for some $P^{\prime} \in \mathrm{T}_{\mathbb{C}} \operatorname{Der}(\log Y)$. Then the claim follows by induction on $d=\operatorname{deg}(P)$.

Example 13. Let $D$ and $Q$ be as in Example 9. Then a Singular GPS05 computation shows that $\pi_{D}$ is injective and that

$$
\sigma(Q) \notin \alpha_{D}\left(\operatorname{Sym}_{\mathcal{O}}^{2} \operatorname{Der}(\log D)\right) .
$$

By Lemma 12 this implies that $Q \notin F_{2} \mathcal{O}[\operatorname{Der}(\log D)]$ and hence, by Example 9 , that $\mathcal{O}[\operatorname{Der}(\log D)] \subsetneq \mathcal{V}_{0}^{D}$.

By the following general statement, injectivity of $\pi_{Y}$ is equivalent to $\mathcal{O}$-torsion freeness of $\operatorname{Sym}_{\mathcal{O}} \operatorname{Der}(\log Y)$.

Lemma 14. Let $R$ be a domain and $M$ a finitely presented torsion free $R$-module. Then the following are equivalent:

(1) $\operatorname{Sym}_{R} M$ is R-torsion free;

(2) $\operatorname{Sym}_{R} M$ is a domain;

(3) $\operatorname{Sym}_{R} M \stackrel{\pi_{M}}{\longrightarrow} \operatorname{Rees}_{R} M$ is injective. 
Proof. Assume that $\operatorname{Sym}_{R} M$ is $R$-torsion free. Let $K=Q(R)$ be the fraction field of $R$. Then $M \otimes_{R} K \cong K^{d}$ where $d=\operatorname{rk}(M)$. By choosing a basis of $K^{d}$ and clearing denominators, one can embed $M \subset R^{d}$. Then

$$
\operatorname{Sym}_{R}(M) \otimes_{R} K \cong \operatorname{Sym}_{R \otimes_{R} K}\left(M \otimes_{R} K\right) \cong \operatorname{Sym}_{K}\left(K^{d}\right)
$$

is a domain, and hence $\operatorname{Sym}_{R} M$ is a domain since $R$ is a domain. Applying $\operatorname{Sym}_{R}$ to the inclusion $M \subset R^{d}$ yields

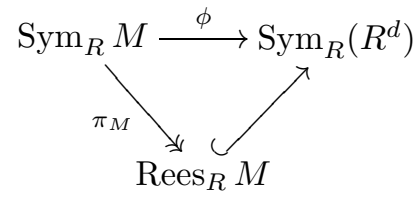

and $\operatorname{ker}(\phi) \otimes_{R} K=0$ since

$$
\operatorname{Sym}_{R}(M) \otimes_{R} K \cong \operatorname{Sym}_{K}\left(K^{d}\right) \cong \operatorname{Sym}_{R}\left(R^{d}\right) \otimes_{R} K .
$$

A presentation

$$
R^{m} \stackrel{\left(a_{i, j}\right)}{\longrightarrow} R^{n} \longrightarrow M \longrightarrow 0
$$

of $M$ defines an isomorphism

$$
\operatorname{Sym}_{R} M \cong R\left[T_{1}, \ldots, T_{n}\right] / J
$$

where $J=\left\langle\sum_{j} a_{i, j} T_{j}\right\rangle$ is a prime ideal since $\operatorname{Sym}_{R} M$ is a domain. Since $\operatorname{Sym}_{R}\left(R^{d}\right)$ is a domain, $\operatorname{ker} \phi$ lifts to a prime ideal $Q \subset R\left[T_{1}, \ldots, T_{n}\right]$. Then $J \subset Q, Q \cap R=0$, and $J \otimes_{R} K=Q \otimes_{R} K$ implies $J=Q$ and hence $\operatorname{ker} \pi_{M}=\operatorname{ker} \phi=0$.

Example 15. Let $D_{4} \subset \mathbb{C}^{4}$ be the central generic hyperplane arrangement defined in Section 5. Then one can compute that the coordinates are zero divisors on $\operatorname{Sym}_{\mathcal{O}}^{2} A_{4}$ at 0. By Lemmata 12, 14, and 24, this implies that $\alpha_{D_{4}}$ is not an isomorphism.

A divisor $D \subset X$ is called Euler homogeneous if locally $\chi(f)=f$ for some $\chi \in \operatorname{Der}(\log D)$ and $f \in \mathcal{O}$ such that $D=(f)$. In this case, $\chi$ is called an Euler vector field and

$$
\operatorname{Der}(\log D) \cong \mathcal{O} \cdot \chi \oplus \operatorname{Ann}_{\Theta}(f) .
$$

If $\operatorname{Der}(\log D) \cong \mathcal{O} \cdot \chi \oplus A$, then $\operatorname{Sym}_{\mathcal{O}} \operatorname{Der}(\log D) \cong \operatorname{Sym}_{\mathcal{O}}(A)[\chi]$. For an Euler homogeneous divisor $D$, this implies that

$$
\operatorname{Sym}_{\mathcal{O}} \operatorname{Der}(\log D) \cong \operatorname{Sym}_{\mathcal{O}}\left(\operatorname{Ann}_{\Theta}(f)\right)[\chi] .
$$

Proposition 16. Let $D \subset X$ be a divisor such that $\operatorname{Sym}_{\mathcal{O}} \operatorname{Der}(\log D)$ is $\mathcal{O}$-torsion free. Then $\mathcal{V}_{0}^{D}=\mathcal{O}[\operatorname{Der}(\log D)]$ follows from

$$
\operatorname{Sym}_{\mathcal{O}} \operatorname{Der}(\log D)=\left(i_{D}\right)_{*} i_{D}^{-1} \operatorname{Sym}_{\mathcal{O}} \operatorname{Der}(\log D) .
$$

If $D$ is Euler homogeneous and $A=\operatorname{Ann}_{\Theta}(f)$ or $A \oplus \mathcal{O} \cdot \chi \cong \operatorname{Der}(\log D)$, then the latter is equivalent to $\operatorname{Sym}_{\mathcal{O}} A=\left(i_{D}\right)_{*} i_{D}^{-1} \operatorname{Sym}_{\mathcal{O}} A$.

Proof. This follows from Corollary [5, Lemmata 11, 12, and 14, and the preceding remarks. 


\section{DEPTH AND TORSION OF SYMMETRIC ALGEBRAS}

Using a theorem of G. Scheja Sch61] on extension of coherent analytic sheaves, we shall give sufficient conditions for $\mathcal{V}_{0}^{D}=\mathcal{O}[\operatorname{Der}(\log D)]$ in terms of the depth and torsion of the symmetric algebras in Proposition 16.

Theorem 17. Let $D \subset X$ be a divisor such that $\operatorname{Sym}_{\mathcal{O}} \operatorname{Der}(\log D)$ is $\mathcal{O}$-torsion free. Let $Z \subset \operatorname{Sing}(D)$ be a closed subset such that $\mathcal{V}_{0}^{D}=\mathcal{O}[\operatorname{Der}(\log D)]$ on $X \backslash Z$. Then $\mathcal{V}_{0}^{D}=\mathcal{O}[\operatorname{Der}(\log D)]$ on $X$ if

$$
\operatorname{depth}_{z}\left(\operatorname{Sym}_{\mathcal{O}}^{k} \operatorname{Der}(\log D)\right) \geq \operatorname{dim}_{z}(Z)+2
$$

for all $z \in Z$ and $k \in \mathbb{N}$. In particular, this holds if $D$ is Euler homogeneous, $A=\operatorname{Ann}_{\Theta}(f)$ or $A \oplus \mathcal{O} \cdot \chi \cong \operatorname{Der}(\log D)$, and

$$
\operatorname{depth}_{z}\left(\operatorname{Sym}_{\mathcal{O}}^{k} A\right) \geq \operatorname{dim}_{z}(Z)+2
$$

for all $z \in Z$ and $k \in \mathbb{N}$.

Proof. This follows from [Sch61, Satz I-III] and Proposition 16.

We shall apply a criterion by C. Huneke Hun81] for the torsion freeness of symmetric algebras.

Proposition 18. Let $R$ be a Noetherian domain and let

$$
0 \longrightarrow R \stackrel{\left(a_{1}, \ldots, a_{m}\right)^{t}}{\longrightarrow} R^{m} \longrightarrow M \longrightarrow 0
$$

be a resolution of $M$. If grade $(I) \geq k+1$ for $I=\left\langle a_{1}, \ldots, a_{m}\right\rangle$, then

$$
\operatorname{depth}\left(I, \operatorname{Sym}_{R}(M)\right) \geq k \text {. }
$$

Proof. We proceed by induction on $k$. By [Hun81, Prop. 2.1], grade $(I) \geq 2$ implies that $\operatorname{Sym}_{R}(M)$ is $R$-torsion free. If $k \geq 2$, then $\operatorname{grade}(I / a) \geq k$ for some $a \in I$ and hence

$$
0 \longrightarrow R / a \stackrel{\left(\left[a_{1}\right], \ldots,\left[a_{m}\right]\right)^{t}}{\longrightarrow}(R / a)^{m} \longrightarrow 0
$$

is a resolution of $M$. Since $\operatorname{Sym}_{R / a}(M / a) \cong \operatorname{Sym}_{R}(M) / a$, the induction hypothesis applies.

Theorem 19. Let $D \subset X$ be an Euler homogeneous divisor and $A=\operatorname{Ann}_{\Theta}(f)$ or $A \oplus \mathcal{O} \cdot \chi \cong \operatorname{Der}(\log D)$. Let $Z \subset \operatorname{Sing}(D)$ be a closed subset such that $\mathcal{V}_{0}^{D}=$ $\mathcal{O}[\operatorname{Der}(\log D)]$ on $X \backslash Z$. For $z \in Z$, let

$$
0 \longrightarrow \mathcal{O}_{z} \stackrel{\left(a_{z, 1}, \ldots, a_{z, m}\right)^{t}}{\longrightarrow} \mathcal{O}_{z}^{m} \longrightarrow A_{z} \longrightarrow 0
$$

be a resolution of $A_{z}$ such that

$$
\operatorname{grade}\left(\left\langle a_{z, 1}, \ldots, a_{z, m}\right\rangle\right) \geq \operatorname{dim}_{z}(Z)+3 \text {. }
$$

Then $\mathcal{V}_{0}^{D}=\mathcal{O}[\operatorname{Der}(\log D)]$ on $X$.

Proof. This follows from Theorem 17, Proposition [18, and [Hun81, Prop. 2.1].

Corollary 20. Let $X$ be a complex manifold of dimension 3 and let $D \subset X$ be a divisor with only isolated quasi-homogeneous singularities. Then $\mathcal{V}_{0}^{D}=\mathcal{O}[\operatorname{Der}(\log D)]$. 
Proof. We may assume that $X \subset \mathbb{C}^{3}$ is an open neighbourhood of $0, D=(f)$ with $f \in \mathcal{O}$, and $\operatorname{Sing}(D)=\{0\}$. Let $x_{1}, x_{2}, x_{3}$ be coordinates on $X$. Then $\partial(f)=\partial_{1}(f), \partial_{2}(f), \partial_{3}(f) \in \mathfrak{m}_{0}$ is an $\mathcal{O}_{0}$-sequence. Hence the Koszul-complex

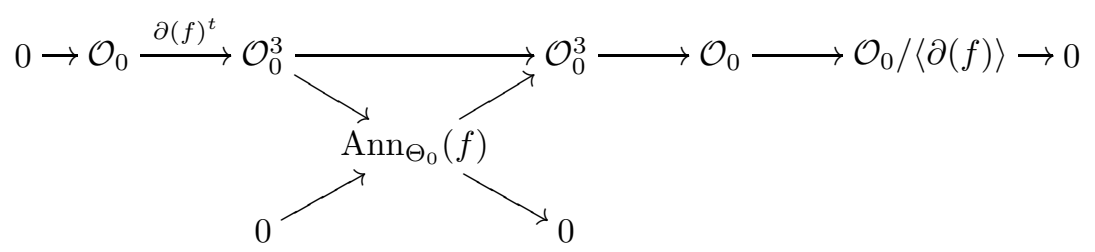

is exact and induces a resolution of $\operatorname{Ann}_{\Theta_{0}}(f)$. Then the claim follows from Theorem 19 with $Z=\operatorname{Sing}(D)$.

Our criterion also applies to some cases of nonisolated singularities.

Example 21. Let $D_{3} \subset \mathbb{C}^{3}$ be the central generic hyperplane arrangement defined in Section 5 Then $D_{3}$ is not a free divisor and has a nonisolated singularity at 0 . By Lemma23 and Proposition 24, $A_{3} \cong \mathcal{O}^{3} / \mathcal{O} \cdot\left(x_{1}, x_{2}, x_{3}\right)$ and $A_{3} \oplus \mathcal{O} \cdot \chi \cong \operatorname{Der}\left(\log D_{3}\right)$. Then, by Examples 1 (2) and 4 (2) on $\operatorname{Sing}\left(D_{3}\right) \backslash\{0\}$ and Theorem 19 for $A=A_{3}$ and $Z=\{0\}, \mathcal{V}_{0}^{D_{3}}=\mathcal{O}\left[\operatorname{Der}\left(\log D_{3}\right)\right]$.

Our approach may fail in dimension $n>3$ even for quasi-homogeneous isolated singularities.

Example 22. Let $x_{1}, x_{2}, x_{3}, x_{4}$ be coordinates on $\mathbb{C}^{4}$ and

$$
f=x_{1}^{2}+x_{2}^{2}+x_{3}^{2}+x_{4}^{2} .
$$

Then $D=(f) \subset \mathbb{C}^{4}$ has a quasi-homogeneous isolated singularity at 0 . One can compute that the coordinates are zero divisors on $\operatorname{Sym}_{\mathcal{O}}^{2} A n_{\mathcal{O}}(f)$ at 0 . By Lemmata 12 and 14 this implies that $\alpha_{D}$ is not an isomorphism.

\section{EXAMPLE OF GENERIC HYPERPLANE ARRANGEMENTS}

We shall provide some background for the examples in the previous sections. Let $x_{1}, \ldots, x_{n}$ be coordinates on $\mathbb{C}^{n}$ and

$$
f_{n}=x_{1} \cdots x_{n}\left(x_{1}+\cdots+x_{n}\right) .
$$

Then $D_{n}=\left(f_{n}\right) \subset \mathbb{C}^{n}$ is a central generic hyperplane arrangement. Let $\chi=$ $\sum_{i} x_{i} \partial_{i}$ be the Euler vector field,

$$
\eta_{i, j}=x_{i} x_{j}\left(\partial_{i}-\partial_{j}\right) \in \operatorname{Der}\left(\log D_{n}\right)
$$

for $i<j$, and $A_{n}=\mathcal{O}\left\langle\eta_{i, j}\right\rangle$. By J. Wiens Wie01, Thm. 3.4],

$$
\operatorname{Der}\left(\log D_{n}\right)=\mathcal{O} \cdot \chi+A_{n}
$$

with a minimal number of generators. Let

$$
\sigma_{i, j, k}=x_{i} \eta_{j, k}-x_{j} \eta_{i, k}+x_{k} \eta_{i, j} \in \operatorname{syz}\left(\eta_{i, j}\right)
$$

for $i<j<k$ and choose a monomial ordering refining $\partial_{1}<\cdots<\partial_{n}$.

Lemma 23. $\left(\eta_{i, j}\right)$ is a standard basis of $A_{n}$ and $\operatorname{syz}\left(\eta_{i, j}\right)=\left\langle\sigma_{i, j, k}\right\rangle$.

Proof. This follows from Buchberger's criterion [GP02, Thm. 1.7.3].

Proposition 24. $\operatorname{Der}\left(\log D_{n}\right)=\mathcal{O} \cdot \chi \oplus A_{n}$. 
Proof. It suffices to verify that no syzygy of $\chi$ and the $\eta_{i, j}$ involves $\chi$. One can obtain the syzygies from a standard basis computation [GP02, Alg. 2.5.4]. The first s-polynomials $x_{k} \chi-\eta_{k, n}$ and $x_{j} \eta_{i, k}-x_{i} \eta_{j, k}$ have a zero $\partial_{n}$ component. Hence only a sequence of s-polynomials starting with $x_{k} \chi-\eta_{k, n}$ can contribute to syzygies involving $\chi$, and the coefficient of $\chi$ remains a monomial. Each element in such a sequence has exactly one monomial involving $x_{n}$. Since the $\partial_{2}, \ldots, \partial_{n-1}$ are leading components of the $\eta_{i, j}$, the sequence terminates with a nonzero element $a_{k} \partial_{1} \equiv x^{\alpha_{k}} \chi \bmod A_{n}$. By the same reason, $\mathcal{O} \cdot \partial_{1} \oplus A_{n}$ is a direct sum and hence $x^{\alpha_{j}} a_{k}=x^{\alpha_{k}} a_{j}$. This implies that the coefficient of $\chi$ in any syzygy is zero.

\section{REFERENCES}

[CM99] Francisco J. Calderón-Moreno, Logarithmic differential operators and logarithmic de Rham complexes relative to a free divisor, Ann. Sci. École Norm. Sup. (4) 32 (1999), no. 5, 701-714. MR.1710757 (2000g:32010)

[CMNM05] Francisco Javier Calderón-Moreno and Luis Narváez-Macarro, Dualité et comparaison sur les complexes de de Rham logarithmiques par rapport aux diviseurs libres, Ann. Inst. Fourier (Grenoble) 55 (2005), no. 1, 47-75. MR2141288 (2006d:32008)

[GP02] Gert-Martin Greuel and Gerhard Pfister, A Singular introduction to commutative algebra, Springer-Verlag, Berlin, 2002, With contributions by Olaf Bachmann, Christoph Lossen and Hans Schönemann, With 1 CD-ROM (Windows, Macintosh, and UNIX). MR 1930604(2003k:13001)

[GPS05] G.-M. Greuel, G. Pfister, and H. Schönemann, Singular 3.0, A Computer Algebra System for Polynomial Computations, Centre for Computer Algebra, University of Kaiserslautern, 2005, http://www.singular.uni-kl.de.

[Hun81] Craig Huneke, On the symmetric algebra of a module, J. Algebra 69 (1981), no. 1, 113-119. MR613861 (82d:13016)

[Meb89] Z. Mebkhout, Le formalisme des six opérations de Grothendieck pour les $D_{X}$-modules cohérents, Travaux en Cours [Works in Progress], vol. 35, Hermann, Paris, 1989, With supplementary material by the author and L. Narváez-Macarro. MR1008245 (90m:32026)

[Sai80] Kyoji Saito, Theory of logarithmic differential forms and logarithmic vector fields, J. Fac. Sci. Univ. Tokyo Sect. IA Math. 27 (1980), no. 2, 265-291. MR586450 (83h:32023)

[Sch61] Günter Scheja, Riemannsche Hebbarkeitssätze für Cohomologieklassen, Math. Ann. 144 (1961), 345-360. MR0148941 (26:6437)

[Tor04] Tristan Torrelli, On meromorphic functions defined by a differential system of order 1, Bull. Soc. Math. France 132 (2004), no. 4, 591-612. MR2131905 (2005m:32015)

[Wie01] Jonathan Wiens, The module of derivations for an arrangement of subspaces, Pacific J. Math. 198 (2001), no. 2, 501-512. MR 1835521(2002d:14090)

Department of Mathematics, Oklahoma State University, 401 MSCS, Stillwater, OKLAHOMA 74078

E-mail address: mschulze@math.okstate.edu 